\title{
Visualization of microscopic stress fields in silica glass in the scanning electron microscope
}

\author{
Giuseppe PEZZoTTI, ${ }^{\dagger}$ Andrea LETO and Alessandro Alan PORPORATI \\ Ceramic Physics Laboratory \& Research Institute for Nanoscience, RIN, Kyoto Institute of Technology, \\ Sakyo-ku, Matsugasaki, 606-8585 Kyoto
}

\begin{abstract}
Quantitative measurements were made in silica glass of highly graded stress fields, as they developed: (i) in the $K$-dominated zone ahead of the tip of a median-type indentation micro-crack; and, (ii) at a silicon-silica interface of a metal-oxide semiconductor (MOS) device. Stress fields could be visualized on a microscopic scale using a field-emission scanning electron microscope (FE-SEM) equipped with a spectrally resolved cathodoluminescence (CL) device, according to piezospectroscopic (PS) assessments. The peculiarity of this newly proposed PS assessment resides in the fact that the performed CL/PS analysis exploited a peculiar luminescence emitted by optically active oxygen point defects in silica glass.

(C2008 The Ceramic Society of Japan. All rights reserved.
\end{abstract}

Key-words : Piezo-spectroscopy, Silica, MOS, Residual stress, Cathodoluminescence

[Received March 31, 2008; Accepted June 19, 2008]

\section{Introduction}

There exist in the literature ${ }^{1-3)}$ a large variety of engineering studies aimed at clarifying reliability issues in silica glass. Great efforts have also been recently made in order to theoretically understand the mechanical behavior of silica glass components when embedded in opto-electronics devices, ${ }^{4)-6)}$ in view of the importance of amorphous silica as a material for dielectric interconnects and films. In silica/silicon MOS devices, residual stresses may arise from the manufacturing process due to differential thermal expansion coefficients as well as polishing and etching procedures; $\left.{ }^{4}\right)$ in addition, during the device operative life, thermal fatigue ${ }^{5)}$ and/or electro-migration processes may also lead to and alteration of the micromechanical state of the device. ${ }^{6}$ Moreover, circuit structures eventually contain cracks and other imperfections at which stresses are further enhanced, with residual stresses driving pre-existing cracks to eventually grow and to ultimately cause the failure of the circuit. All these factors and circumstances may greatly reduce the operation lifetime of MOS devices.

At the present stage of technology development and in view of the ongoing process of miniaturization of MOS devices, it has become of fundamental importance to develop an advanced analytical tool, which could be helpful in clarifying the mechanics of fatigue/stress-related problems. However, residual stresses, despite their significance and importance in modern devices, cannot be visualized on a microscopic scale by means of conventional microscopy techniques, especially when they are highly graded in their spatial distribution and/or stored in very small structures (i.e., as it is the case in MOS devices).

In this paper, we show a CL/PS approach to analyze nondestructively areas of stress intensification in the silica glass comprised in MOS devices; we have recently physically substantiated and applied this new analytical practice ${ }^{7,8)}$ in the belief that it will help device designers to formulate quantitative criteria for

\footnotetext{
Corresponding author: G. Pezzotti; E-mail: pezzotti@chem.kit. ac.jp
}

the analysis of interface cracking (and related growth rate) and, ultimately, to precisely predict its criticality for device lifetime. The adopted strategy is based on quantitative monitoring CL spectral shifts on selected bands with respect to their unstressed (reference) state. A fundamental advantage of the CL/PS technique is that a low-energy electron beam is capable to stimulate luminescence from very localized areas, typically on a submicrometer scale. Furthermore, improvements in spatial resolution can be also achieved upon applying deconvolution procedures for the electron probe. This circumstance represents, as we shall extensively show in the remainder of this paper, a real break-trough in quantitatively assessing highly graded (residual and applied) stress distributions in silica glass and related devices.

\section{Experimental procedures}

Non-stoichiometric carbon-doped $\mathrm{SiO}_{x}$ (henceforth simply referred to as $\mathrm{C}$-doped $\mathrm{SiO}_{x}$ ) was obtained from a large glass pool representing the interlayer dielectric material of a wide $\mathrm{Cu}$ /Ta-interconnect pattern belonging to a model electronic chip (Intel Corp., Santa Clara, CA). The $\mathrm{SiO}_{x} / \mathrm{Si}$ interface studied as an application of the PS technique belonged to an intermediate unit of a MOS model chip (Matsushita Technoresearch, Kadoma, Osaka, Japan) prepared by chemical vapor deposition. Prior to analysis, a $2 \mathrm{~nm}$ thick layer of $\mathrm{Pt}$ was deposited on both samples to avoid charging induced by electron beam irradiation.

In the CL spectrum of high-purity silica glass (Fig. 1), the predominant band is usually observed in the red region at about 630 $\mathrm{nm}$ ( $R$-band, henceforth). This band is related to non-bridging oxygen hole centers (NBOHC), namely to oxygen excess sites. ${ }^{9)}$ An additional band in the blue region at about $460 \mathrm{~nm}$ (band $B$, henceforth) arises from a population of two-fold coordinated silicon centers, generated in the presence of an oxygen vacancy site (usually referred to as oxygen deficient centers, ODC). ${ }^{10)}$ The structure and related electronic transitions, involved with these two types of defective cluster in the $\mathrm{SiO}_{x}$ network, are schematically drawn in Fig. 1. A yellow $(Y)$ band arises from the presence of structural water, ${ }^{10)}$ while the ultraviolet band at about 290 

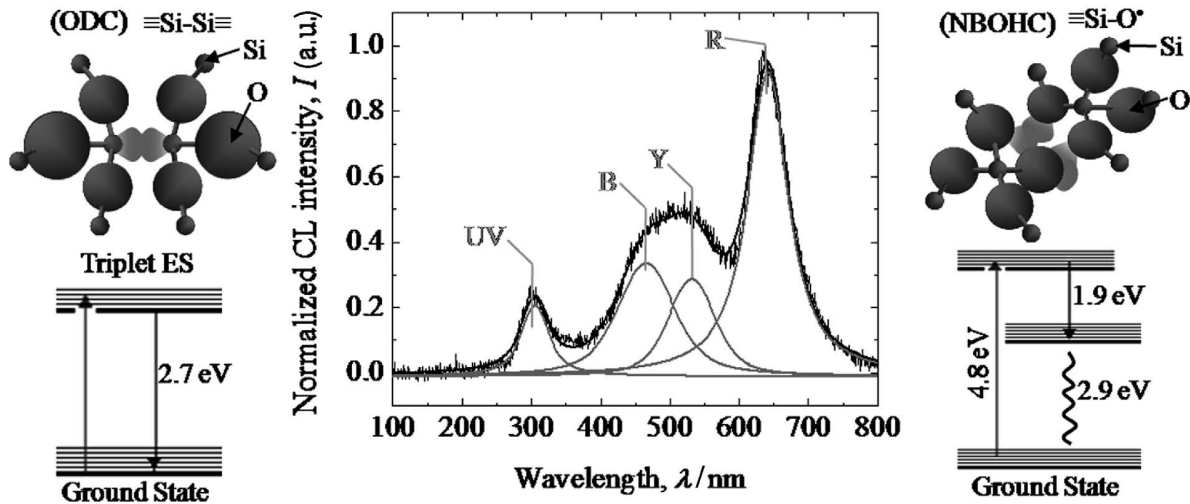

Fig. 1. CL spectrum of high-purity silica glass. The structure and related electronic transitions, involved with non-bridging oxygen hole centers (NBOHC) and the oxygen deficient centers, ODC, are schematically drawn.

nm ( $U V$ band, henceforth) originates from trivalent silicon centers and/or from other point-defects generated by electron beam irradiation. ${ }^{10)}$ The CL spectrum of C-rich silica is characterized by a relatively low intensity of the $R$ band, as a consequence of this band being related to oxygen-excess centers and thus conspicuously annihilated in the presence of dangling bonds.

In amorphous materials, the in-plane probe response function (PRF) for electrons is fully symmetric and can be represented according to a Gaussian equation, as follows:

$$
G\left(x, y ; x_{0}, y_{0} ; V\right)=C_{1} \exp \frac{-\left(x-x_{0}\right)^{2}}{C_{2} V^{n}} \times \exp \frac{-\left(y-y_{0}\right)^{2}}{C_{2} V^{n}}
$$

where, $V$ is the acceleration voltage of the impinging electrons, and the abscissas, $(x, y)$ and $\left(x_{0}, y_{0}\right)$ represent any point in the focal plane and the geometrical center of the focused electron beam, respectively. The PRF of silica glass was experimentally retrieved as a function of acceleration voltage from a line scans across an atomically sharp interface between $\mathrm{SiO}_{x}$ and silicon, as it has been shown in detail in a previous paper. ${ }^{7)}$ The parameter, $C_{1}$, was taken equal to unity after CL intensity normalization, and the parameters $C_{2}$ and $n$ were experimentally determined as 0.022 and 1.05 , respectively, according to a bestfitting procedure of experimental data. Figure 2(a) shows the experimentally determined electron PRF for amorphous $\mathrm{SiO}_{x}$ as recorded at different acceleration voltages, $6 \leq V \leq 10 \mathrm{kV}$. Note that the choice of the same constant $C_{2}$ along $x$ and $y$ directions corresponds to the assumption that no in-plane preferential orientation arises for an amorphous structure both regarding to electron diffusion and light scatter. Based on the experimental knowledge of the PRF, an in-plane probe deconvolution procedure can be attempted to restore the collected stress data from the
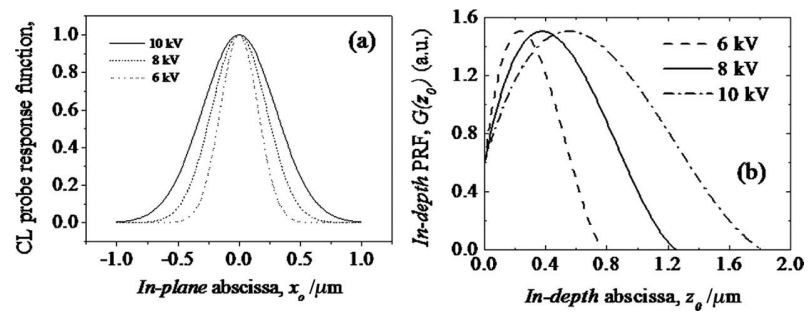

Fig. 2. (a) Experimentally determined in-plane electron probe response function (PRF) for amorphous $\mathrm{SiO}_{x}$ as recorded at different acceleration voltages; (b) calculated in-depth PRF for amorphous silica. effect of the finite size of the probe.

When the CL characterization is aimed at the determination of distribution profiles with a strong three-dimensional character (i.e., with non-negligible components along the in-depth zdirection), a different type of PRF, as given by Donolato, ${ }^{11}$ should be used. The differential contribution of each portion of volume of the luminescent probe (i.e., the PRF) was then obtained from calculating the first derivative of the normalized $\mathrm{CL}$ intensity. Experimental curves collected for silica glass as a function of the abscissas, $z_{\mathrm{o}}$, are given for different voltages in Fig. 2(b).

Throughout the present CL characterization, an acceleration voltage in the range, $6 \leq V \leq 10 \mathrm{kV}$, was selected, which was well above the detection limit of the spectrum of $\mathrm{SiO}_{x}$ in our CL device $(V \approx 3 \mathrm{kV})$. This choice was made with a partial sacrifice of beam sharpness, because it allowed us to obtain a reliable and stable spectrum.

Stress assessments according to the PS behavior of the NBOHC band of $\mathrm{SiO}_{x}$ are possible, provided that it can be neatly separated from the rest of the CL spectrum, especially from additional bands in the yellow region, as explained in a previous paper. ${ }^{10)}$ Precise characterizations of the spectral shift rate with applied stress have been shown elsewhere, ${ }^{7)}$ according to different calibration methods. The shift rate, which is usually referred to as the PS coefficient, is a material property. For the NBOHC band as displayed in the $\mathrm{SiO}_{x}$ spectrum of high-purity and Cdoped silica glasses, the PS coefficient has been determined as shown in Table 1.9)

Table 1. CL-PS Coefficients for the NBOHC Band of High-Purity and C-doped Silica Glasses

\begin{tabular}{lcc}
\hline & \multicolumn{2}{c}{ PS Coefficients $(\mathrm{nm} / \mathrm{GPa})$} \\
\cline { 2 - 3 } & Uniaxial $\Pi$ & Biaxial $\Pi$ \\
\hline High-purity $\mathrm{SiO}_{x}$ & $-8.9 \pm 0.3$ & $-17.2 \pm 0.4$ \\
$C$-doped $\mathrm{SiO}_{x}$ & $/$ & $-13.8 \pm 0.5$ \\
\hline
\end{tabular}

\section{Results and discussion}

\subsection{Stress field developed ahead of an indentation crack}

Similar to the case of interstitial impurity centers, the PS behavior of bands arising from oxygen point defects can be 
assumed to obey the Grabner's formalism. ${ }^{13)}$ According to this formalism, the stress state in a material is related to the wavelength shift (with respect to its unstressed state), $\Delta \lambda$, of a selected luminescence band, according to the following tensorial equation:

$$
\begin{gathered}
\Delta \lambda=\left(\begin{array}{lll}
\Pi_{11} & \Pi_{12} & \Pi_{13} \\
\Pi_{21} & \Pi_{22} & \Pi_{23} \\
\Pi_{31} & \Pi_{32} & \Pi_{33}
\end{array}\right):\left(\begin{array}{ccc}
\sigma_{11} & \tau_{12} & \tau_{13} \\
\tau_{21} & \sigma_{22} & \tau_{23} \\
\tau_{31} & \tau_{32} & \sigma_{33}
\end{array}\right)^{T}=\Pi_{i j} \sigma_{i j} \\
=\left(\begin{array}{ccc}
\Pi & 0 & 0 \\
0 & \Pi & 0 \\
0 & 0 & \Pi
\end{array}\right):\left(\begin{array}{ccc}
\sigma_{11}^{*} & 0 & 0 \\
0 & \sigma_{22}^{*} & 0 \\
0 & 0 & \sigma_{33}^{*}
\end{array}\right)^{T}=\Pi \sigma_{i i}^{*}
\end{gathered}
$$

where, $i, j=1,2,3=x, y, z ; \Delta \lambda=\lambda-\lambda_{0}$, is the wavelength shift observed with respect to the unstressed wavelength, $\lambda_{0}$; the repetitive index notation is applied to a general stress state, $\sigma_{i j}$, and, $\Pi_{i j}$, is the second-rank tensor of PS coefficients. An asterisk to the stress components denotes the choice of a set of Cartesian axes coincident with the principal stress directions. Note that the PS tensor in the silica amorphous network degenerates into a scalar quantity, the PS coefficient, П. According to Eq. (2), the spectral shift is an invariant with respect to the selected system of coordinates and is related by direct proportionality to the algebraic sum of the principal components of the stress tensor (as reflected by the repetitive index used in the right hand of Eq. (2)), and thus to its trace.

The surface stress field developed ahead of the tip of a plane crack propagating along the in-plane direction $x$ can be given as: ${ }^{14)}$

$$
\begin{aligned}
& \left.\sigma_{x x}=\frac{K_{\mathrm{I}}}{\sqrt{2 \pi r}} \cos \frac{\theta}{2}\left(1-\sin \frac{\theta}{2} \sin \frac{3 \theta}{2}\right)\right] \\
& \left.\sigma_{y y}=\frac{K_{\mathrm{I}}}{\sqrt{2 \pi r}} \cos \frac{\theta}{2}\left(1+\sin \frac{\theta}{2} \sin \frac{3 \theta}{2}\right)\right\}+ \text { higher-order terms. } \\
& \tau_{x y}=\tau_{y x}=\frac{K_{\mathrm{I}}}{\sqrt{2 \pi r}} \sin \frac{\theta}{2} \cos \frac{\theta}{2} \cos \frac{3 \theta}{2}
\end{aligned}
$$

where, $K_{\mathrm{I}}$ is the crack-tip stress intensity factor and $(r, \theta)$ are polar coordinates, as depicted in Fig. 3(a). It should be noted that the shear stress component, $\tau_{x y}$, is zero at any location straight ahead of the crack tip along the abscissa, $r$. In addition, according to the Grabner's formalism, the observed spectral shift is only affected by the principal stress components. Upon translating the stress tensor, as given by Eq. (3), into principal stress components, and upon neglecting the higher-order terms, Eq. (3) can be rewritten at any location straight ahead of the crack tip, as follows:

$$
\begin{aligned}
\Delta \lambda(r) & =\Pi \sigma_{i i}^{*}=\Pi \frac{K_{\mathrm{I}}}{\sqrt{2 \pi r}} \cos \frac{\theta}{2}\left|\begin{array}{ccc}
1+\sin \frac{\theta}{2} & 0 & 0 \\
0 & 1-\sin \frac{\theta}{2} & 0 \\
0 & 0 & 0
\end{array}\right| \\
& =2 \Pi \frac{K_{\mathrm{I}}}{\sqrt{2 \pi r}} \cos \frac{\theta}{2}
\end{aligned}
$$

Equation 4 gives us a chance to experimentally locate the crack-tip stress intensity factor, $K_{\mathrm{I}}$, in silica glass through a PS approach. However, in the experimental practice, the wavelength shift (with respect to an unstressed location) collected by the CL probe represents a Gaussian convolution (i.e., through the inplane PRF given by Eq. (1)) of the highly graded crack-tip stress field into the CL probe volume. Therefore, a direct determination of the parameter $K_{\mathrm{I}}$ shall involve the appropriate consideration of the probe Gaussian filter. Note that here we neglect any spectral shift contribution from the in-depth abscissa, $z$, the rationale for doing so resides in the negligible size of the electron probe $(<1$ $\mu \mathrm{m})$ as compared to the sub-surface depth of the (median) indentation crack. The Gaussian convoluted spectral shift, $\Delta \lambda\left(x_{0}, y_{0} ; V\right)$, in the plane of polish can be then represented, as follows:

$$
\begin{aligned}
& \overline{\Delta \lambda}\left(x_{0}, y_{0} ; V\right)=\frac{\int_{-\infty}^{+\infty} \int_{-\infty}^{+\infty} G\left(x, x_{0} ; y, y_{0} ; V\right) \Delta \lambda(x, y) \mathrm{d} x \mathrm{~d} y}{\int_{-\infty}^{+\infty} \int_{-\infty}^{+\infty} G\left(x, x_{0} ; y, y_{0} ; V\right) \mathrm{d} x \mathrm{~d} y} \\
& =2 \Pi K_{I} \frac{\int_{0}^{+\infty} \int_{-\pi}^{\pi} \sqrt{\frac{r}{2 \pi}} \cos \left(\frac{\theta}{2}\right) \times \exp \left[-\frac{\left(r^{2}+x_{0}^{2}\right)}{C_{2} V^{n}}\right] \times \exp \left(\frac{2 r x_{0} \cos \theta}{C_{2} V^{n}}\right) \mathrm{d} r \mathrm{~d} \theta}{\int_{0}^{+\infty} \int_{-\pi}^{\pi} r \times \exp \left(-\frac{r^{2}+x_{0}^{2}-2 r x_{0} \cos \theta}{C_{2} V^{n}}\right) \mathrm{d} r \mathrm{~d} \theta} \\
& =\Pi \bar{\sigma}_{i i}^{*}\left(x_{0}, y_{0} ; V\right)
\end{aligned}
$$

where $\bar{\sigma}_{i i}^{*}\left(x_{0}, y_{0} ; V\right)$ is the convoluted trace of the principal stress tensor ahead of the crack tip. Equation (5) can be used to determine the stress intensity factor, $K_{\mathrm{I}}$, from a procedure of best fitting of the experimentally determined profile of spectral shifts ahead of the crack tip. A set of experimental data collected on
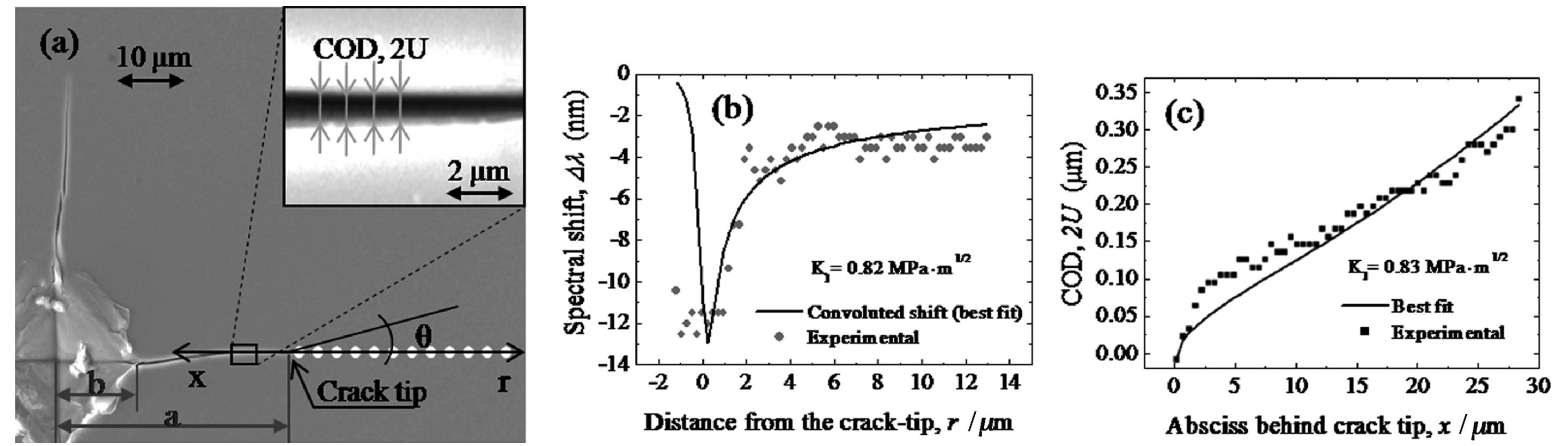

Fig. 3. (a) high-resolution FE-SEM images of a Vickers indentation and, in the inset, a schematic of the crack opening displacement (COD) calculation; (b) experimentally determined profile of spectral shifts ahead of the crack tip; (c) plot of the COD for a median indentation crack propagated in the $\mathrm{SiO}_{x}$ material. 
amorphous $\mathrm{SiO}_{x}$ and the results of its best fitting, according to Eq. (5), are shown in Fig. 3(b). The procedure locates a $K_{\mathrm{I}}$ value of $0.82 \mathrm{MPa} \cdot \mathrm{m}^{1 / 2}$. In order to confirm the precision of the results found by the CL/PS procedure, the crack-tip stress intensity factor, $K_{\mathrm{I}}$, of the median indentation crack in Fig. 3(a) was also measured according to a quantitative assessment of crack opening displacements (COD), $2 U(x)$, from high-resolution FE-SEM images (cf. inset of Fig. 3(a)), according to the procedure first suggested by Roedel and coworkers. ${ }^{15)}$ For a median crack configuration, $K_{I}$ and $U(x)$ can be related through the following equation: ${ }^{16)}$

$$
U(x)=\frac{4 K_{\mathrm{I}}}{\pi E^{\prime}} Y\left(\frac{x}{a}\right)
$$

where, the weight function, $Y\left(\frac{x}{a}\right)$, for an indentation median
crack is given by: ${ }^{17)}$

$$
Y\left(\frac{x}{a}\right)=\sqrt{b}\left[\sqrt{A\left(\frac{x}{a}\right)}+\sqrt{B^{3}\left(\frac{x}{a}\right)}+\sqrt{C^{5}\left(\frac{x}{a}\right)}\right]
$$

where $x$ is an abscissa taken along the crack path (with an opposite direction to the abscissa, $r$, of crack propagation), and the numerical parameters $A, B$, and $C$ are given, as follows:

$$
\left.\begin{array}{c}
A=\sqrt{\frac{\pi a}{2 b}} \\
B \cong 0.011+1.8197 \ln \left(\frac{a}{b}\right) \\
C \cong-0.6513+2.121 \ln \left(\frac{a}{b}\right)
\end{array}\right\}
$$

with $a$ being the distance between the crack tip and the center of the indentation print, and $b$ half the diagonal of the indentation print (cf. Fig. 3(a)). E' is the plane stress Young's modulus of the silica material (taken as: $E^{\prime}=78 \mathrm{GPa}$ ). A plot of the COD for a median indentation crack propagated in the $\mathrm{SiO}_{x}$ material is shown in Fig. 3(c). The stress intensity obtained from leastsquare fitting of the experimental COD data (i.e., according to Eqs. (7) and (8)) was $K_{\mathrm{I}}=0.83 \mathrm{MPa} \cdot \mathrm{m}^{1 / 2}$. This result, which was obtained by an independent experimental approach on the same crack, is in good agreement with the $K_{\mathrm{I}}$ obtained by the PS method, thus confirming the actual possibility of directly assessing stress fields in silica glass in the FE-SEM.

\subsection{Stress intensification at a $\mathrm{Si} / \mathrm{SiO}_{x}$ interface}

Figure 4(a) is a FE-SEM micrograph taken at the location of a sharp $\mathrm{SiO}_{x} / \mathrm{Si}$ interface in correspondence of an intermediate unit in a CVD-prepared MOS device. Figures 4(b) and (c) show two typical CL spectra, one collected with the electron beam placed at a location far away from the interface and the other in correspondence of the $\mathrm{SiO}_{x} / \mathrm{Si}$ interface ((b) and (c), respectively). As can be easily seen, the morphological difference between the two CL spectra in Fig. 4(b) and (c) is significant. The salient features of such difference can be summarized as follows: (i) the intensity of the $B$ band with respect to the $R$ band is conspicuously increasing with approaching the Si interface, suggesting that the concentration of oxygen-excess defects is dramatically reduced in the neighborhood of the $\mathrm{SiO}_{x} / \mathrm{Si}$ interface; and, (ii) although no carbon was intentionally added to the silica of this sample, the relative intensity trend suggests that aliovalent impurities (i.e., including carbon) tend to segregate to the $\mathrm{SiO}_{x} / \mathrm{Si}$ interface forming dangling bonds.

Several line scans were collected at different acceleration voltages $(6$ and $8 \mathrm{kV})$ along selected lines from the silica side perpendicular to the $\mathrm{SiO}_{x} / \mathrm{Si}$ interface, as shown by dots in Fig. 4(a). In the series of CL spectra collected at different acceleration voltages, we monitored the $R$ band with respect to its spectral shift. Band shifts were then converted into trace values of convoluted stress tensor according to the PS coefficient and plotted in Fig. $\mathbf{5}$, as a function of distance, $x$, from the interface. Upon decreasing acceleration voltage, and thus the CL probe size, the stress magnitude at the $\mathrm{SiO}_{x} / \mathrm{Si}$ interface increased. This trend can be qualitatively explained by considering that a quite steep stress gradient should exist in proximity of the interface. However, a quantitative analysis of the residual stress field piled up in correspondence of the $\mathrm{SiO}_{x} / \mathrm{Si}$ interface requires an in-depth probe deconvolution procedure.

A tensorial description of the residual stress field, developed in the presence of fully embedded patterns, has been given by $\mathrm{Hu},{ }^{18)}$ according to the Green's function approach. For the $i$-th rectangular inclusion, belonging to a regular array of $N$ inclusions with infinite length (along a Cartesian $x$-axis) but finite width and depth, embedded in a half-space (which was the case here), the components of the stress tensor can be given as follows:
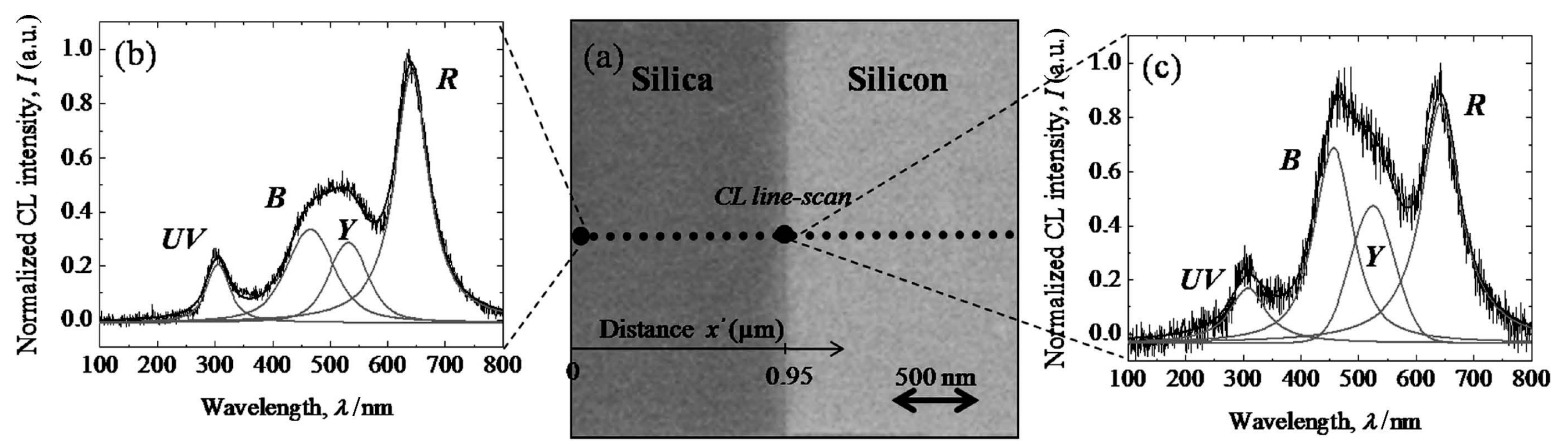

Fig. 4. FE-SEM micrograph taken at the location of a sharp $\mathrm{SiO}_{x} / \mathrm{Si}$ interface in correspondence of an intermediate unit in a CVD-prepared MOS device; (b) typical CL spectra collected with the electron beam placed at a location far away from the interface; (c) typical CL spectra collected in correspondence of the $\mathrm{SiO}_{x} / \mathrm{Si}$ interface. 


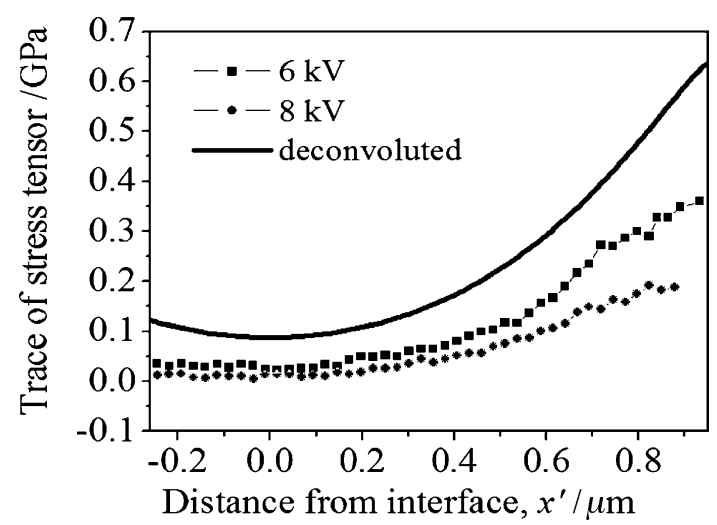

Fig. 5. Experimental residual stress obtained for 6 and $8 \mathrm{kV} \mathrm{CL}$ line scans, and the deconvoluted profile of the trace of the stress tensor, $\sigma_{i i}^{*}$.

$$
\sigma_{x x}=-\frac{2 F}{\pi} \sum_{i=1}^{N}\left\{\frac{\left[\Psi+\frac{w}{2}\right]^{3}}{\left[\left(\Psi+\frac{w}{2}\right)^{2}+z^{2}\right]^{2}}-\frac{\left[\Psi-\frac{w}{2}\right]^{3}}{\left[\left(\Psi-\frac{w}{2}\right)^{2}+z^{2}\right]^{2}}\right\}
$$

$$
\sigma_{z z}=-\frac{2 F}{\pi} z^{2} \sum_{i=1}^{N}\left\{\frac{\left[\Psi+\frac{w}{2}\right]}{\left[\left(\Psi+\frac{w}{2}\right)^{2}+z^{2}\right]^{2}}-\frac{\left[\Psi-\frac{w}{2}\right]}{\left[\left(\Psi-\frac{w}{2}\right)^{2}+z^{2}\right]^{2}}\right\}
$$

$$
\tau_{x z}=-\frac{2 F}{\pi} z \sum_{i=1}^{N}\left\{\frac{\left[\Psi+\frac{w}{2}\right]^{2}}{\left[\left(\Psi+\frac{w}{2}\right)^{2}+z^{2}\right]^{2}}-\frac{\left[\Psi-\frac{w}{2}\right]^{2}}{\left[\left(\Psi-\frac{w}{2}\right)^{2}+z^{2}\right]^{2}}\right\}
$$

where, $w$ is the width of the Si islands and our choice of Cartesian axes was with the origin taken at the center of the pattern, as shown in Fig. 4(a). The geometrical parameter, $\Psi$, is given as:

$$
\Psi=x-N(w+d)
$$

where, $d$ is the width of the $\mathrm{SiO}_{x}$ islands (of depth, $t$ ) and $F$ is a term which takes into consideration the mismatches in thermal expansion and elastic constants between different phases, as well as the difference in temperature excursion during manufacturing from a high-temperature stress-free state. Note, however, that unlike the problem studied in the previous section, the in-depth size of the electron probe is in this case comparable to the distance over which the residual stress gradient develops along the $z$ direction. In addition, as previously discussed, the output of the PS assessment is a scalar quantity, namely a spectral shift, which should be then related to the convoluted trace of the principal stress tensor. The Grabner's tensorial equation for the stress state nearby the interface can be re-written, as follows:

$$
\begin{aligned}
& \frac{\Delta \lambda}{\Pi}=T \sigma_{i j} T^{-1}=\sigma_{i i}^{*}=\left|\begin{array}{ccc}
\cos \psi & 0 & \sin \psi \\
0 & 1 & 0 \\
-\sin \psi & 0 & \cos \psi
\end{array} \| \begin{array}{ccc}
\sigma_{x x} & 0 & \tau_{x z} \\
0 & 1 & 0 \\
\tau_{z x} & 0 & \sigma_{z z}
\end{array}\right| \begin{array}{ccc}
\cos \psi & 0 & -\sin \psi \\
0 & 1 & 0 \\
\sin \psi & 0 & \cos \psi
\end{array} \mid \\
& =\left|\begin{array}{ccc}
\sigma_{x x}^{*} & 0 & 0 \\
0 & 0 & 0 \\
0 & 0 & \sigma_{z z}^{*}
\end{array}\right|=\left|\begin{array}{ccc}
\frac{\sigma_{x x}+\sigma_{z z}}{2}+\Lambda \frac{\sigma_{x x}-\sigma_{z z}}{2} & 0 & 0 \\
0 & 0 & 0 \\
0 & 0 & \frac{\sigma_{x x}+\sigma_{z z}}{2}-\Lambda \frac{\sigma_{x x}-\sigma_{z z}}{2}
\end{array}\right| \\
& =\sigma_{x x}^{*}+\sigma_{z z}^{*}
\end{aligned}
$$

where, $T$ and $T^{-1}$ are transformation matrices, $\Psi$ is the rotation angle for the Cartesian system, and the parameter, $\Lambda$, is computed as follows:

$$
\Lambda=\cos \left[\arctan \left(\frac{2 \tau_{x z}}{\sigma_{x x}-\sigma_{z z}}\right)\right]+\tau_{x z} \sin \left[\arctan \left(\frac{2 \tau_{x z}}{\sigma_{x x}-\sigma_{z z}}\right)\right]
$$

where, $\sigma_{x y}, \sigma_{z z}$, and $\tau_{x z}$ are given by Eqs. (9), (10), and (11), respectively.

The profile of convoluted spectral shifts can be then expressed according to the following integral equation:

$$
\overline{\Delta \lambda}\left(x_{0}, z_{0} ; V\right)=\frac{\int_{-\frac{d}{2}}^{\frac{d}{2}} \int_{0}^{t} G\left(x, x_{0} ; z, z_{0} ; V\right) \Delta \lambda(x, z) \mathrm{d} x \mathrm{~d} z}{\int_{-\frac{d}{2}}^{\frac{d}{2}} \int_{0}^{t} G\left(x, x_{0} ; z, z_{0} ; V\right) \mathrm{d} x \mathrm{~d} z}=\Pi \bar{\sigma}_{i i}^{*}\left(x_{0}, z_{0} ; V\right)
$$

where, the trace of the residual stress tensor, $\bar{\sigma}_{i i}^{*}$, is expressed by Eq. (13). In other words, the bias involved with the finite size of the probe can be taken into consideration and corrected by exploiting the knowledge of the PRF of the material. Equation (15) can be iteratively solved in search for the value of the parameter $F$, displayed in Eqs. (9)-(12), for which the convoluted shift profile best fits, at any location, the experimentally observed spectral shift distribution. Once the $F$ value has been determined, the deconvoluted residual stress distribution stored in the amorphous $\mathrm{SiO}_{x}$ material in the neighborhood of the $\mathrm{SiO}_{x} / \mathrm{Si}$ interface can be plotted according to Eqs. (9)-(12). The deconvoluted profile of the trace of the stress tensor, $\sigma_{i i}^{*}$, is shown in Fig. 5. This latter plot represents our desired goal of a quantitative evaluation of residual stresses at a $\mathrm{SiO}_{x} / \mathrm{Si}$ interface using an electron probe.

\section{Conclusion}

In this paper, we have shown the possibility of performing quantitative assessments of residual stresses in amorphous silica on a microscopic scale using a FE-SEM. The Grabner's formalism has been invoked to explain CL wavelength shifts observed in the $K$-dominated zone at the tip of an indentation crack and in the amorphous silica side of a MOS device nearby a $\mathrm{SiO}_{x} / \mathrm{Si}$ interface. The present experimental PS assessments, substantiated by theoretical arguments, take advantage of the microscopic size and scanning flexibility of a low-energy electron probe. The PS experimental method does not require sample manipulation and is minimally intrusive; moreover, it has a potential for becoming a routine practice for the direct in-situ assessment of electronic devices. Spatial deconvolution procedures have also been proposed for both in-plane and in-depth stress distributions, which may enable one to minimize the error related to the finite 
volume of the probe, thus experimentally obtaining the actual stress profiles. The deconvolution procedures shown in this paper, which can be easily generalized to perform more complicated stress analyses of silica glass, give for the first time glass scientists and technologists the possibility "to visualize" the weak points in a glass structure before fracture actually occurs.

\section{References}

1) Z. Suo, "Interfacial and Nanoscale Failure - Comprehensive Structural Integrity," 8, Elsevier Science Ltd., Amsterdam (2003) pp. 265-324.

2) Y. Hibino and H. Hanafusa, J. Non-Cryst. Solids., 107(1), 2326 (1988).

3) I. De Wolf, M. Ignat, G. Pozza, L. Maniguet and H. E. Maes, J. Appl. Phys., 85, 6477-6485 (1999).

4) Q. Ma, S. Chiras, D. R. Clarke and Z. Suo, J. Appl. Phys., 78, 1614-1622 (1995).

5) I. De Wolf, Semicond. Sci. Technol., 11, 139-154 (1996).

6) K. Pinardi, S. C. Jain, M. Willander, A. Atkinson, H. E. Maes and D. Van Overstraeten, J. Appl. Phys., 84, 2507-2512 (1998).

7) A. Leto, A. A. Porporati, W. Zhu, M. Green and G. Pezzotti,
J. Appl. Phys., 101, 093514 (2007).

8) A. Leto, M. C. Munisso, A. A. Porporati, W. Zhu and G. Pezzotti, J. Phys. Chem. A, 18380491 (2008).

9) L. Skuja, J. Non-Cryst. Solids, 179, 51-69 (1994).

10) H. J. Fitting, T. Barfels, A. N. Trukhin and B. Schmidt, J. NonCryst. Solids, 279, 51-59 (2001).

11) C. Donolato and P. Venturi, Phys. Stat. Sol. (a), 73, 377-387 (1982).

12) K. Kanaya and S. Okayama, J. Phys. D. Appl. Phys., 5, 42-49 (1972).

13) L. Grabner, J. Appl. Phys., 49, 580-583 (1978).

14) W. Zhu, A. A. Porporati, A. Matsutani, N. Lama and G. Pezzotti, J. Appl. Phys., 101, 103531 (2007).

15) J. Roedel, J. Kelly and B. R. Lawn, J. Am. Ceram. Soc., 73, 3313 (1990).

16) H. Tada, P. C. Paris and G. R. Irwin, "The Stress Analysis of Cracks," Handbook Del. Research Corporation, Hellertown, PA (1973).

17) T. Fett, "Computation of the Crack Opening Displacements for Vickers Indentation Cracks," Report No. FZKA 6757, Forschungszentrum Karlsruhe, Karlsruhe, Germany (2002).

18) S. M. Hu, J. Appl. Phys., 70, R53-R80 (1991). 